Original Article (short paper)

\title{
The validity of Keiser-M3 stationary bicycle with standard ergometer for physiological measurements associated with maximum effort
}

\author{
Rousseau S. Veiga $^{1,2}$ (D), Camila B. Müller ${ }^{1,2}$ (D), Léo D. Cabistany ${ }^{1,2}$ (D), \\ ${\text { Andressa C. } \text { Formalioni }^{3} \text { (D), Eraldo S. Pinheiro }}^{1,2}$ (D), Fabrício B. Del Vecchio ${ }^{1,2}$ (D) \\ ${ }^{1}$ Escola Superior de Educação Física, Pelotas, RS, Brasil. \\ ${ }^{2}$ Universidade Federal de Pelotas, Pelotas, RS, Brasil. \\ ${ }^{3}$ Universidade de São Paulo, São Paulo, SP, Brasil.
}

\begin{abstract}
Aim: This study aims to investigate the concordance between two cycle ergometers for variables measured in the test of maximum incremental effort. Methods: This correlation study enrolled 15 inactive women $(19,2 \pm$ 4,0 years old). At random, the participants performed two maximal effort incremental tests (MEIT), using cycle ergometers (Keiser-M3 and Ergo-167) on different days with a minimum interval of 72 hours. The test had stages of two minutes, and two-step increments were carried out at the end of each stage. Lactate concentration ([LAC]), and rated perceived exertion (RPE) were collected, in addition to monitoring oxygen uptake $\left(\mathrm{VO}_{2}\right)$ and heart rate (HR). The Paired t-test was carried out to compare physiologic variables, Lin's test was used for correlation, and Bland Altman was used to measuring concordances among variables. Results: The correlations between physiologic parameters were considered strong for HR on the anaerobic threshold (Keiser M3 = $146 \pm 14 \mathrm{bpm}$; Ergo-167 = $149 \pm 9 \mathrm{bpm} ; \mathrm{r}=0.762$ ), greater blood lactate value (Keiser M3 = 9,97 $\pm 2,51 \mathrm{mmol} / \mathrm{L} ;$ Ergo-167 $=9.71 \pm 2.56 \mathrm{mmol} / \mathrm{L} ; \mathrm{r}=0.820), \mathrm{VO}_{2 \max }$ $($ Keiser M3 = 38,37 $\pm 6,97 \mathrm{~mL} / \mathrm{kg} / \mathrm{min}$; Ergo-167 $=36.06 \pm 8.4 \mathrm{~mL} / \mathrm{kg} / \mathrm{min} ; \mathrm{r}=0,806)$ and $\mathrm{HR}_{\max }($ Keiser M3 $=186 \pm$ $53 \mathrm{bpm}$; Ergo-167 = $188 \pm 11 \mathrm{bpm} ; \mathrm{r}=0.716$ ). Conclusion: The results show the feasibility of using the cycle ergometer Keiser, model M3, to perform the maximal effort incremental test, whereas there was high concordance in the physiologic responses in both ergometers for inactive women.
\end{abstract}

Keywords: validity study, exercise, incremental test, lactate threshold, oxygen uptake.

\section{Introduction}

Incremental tests were performed in different contexts since clinical and functional variables measured at rest are not capable to adequately identify an individual's ability to perform physical exercises ${ }^{1,2}$. The results from these tests are used to obtain relevant information to the prevention and treatment of pathological conditions ${ }^{3}$, as well as for prescribing and understanding the physiological adjustments associated with physical training ${ }^{4}$.

In order to assess maximal oxygen uptake $\left(\mathrm{VO}_{2 \max }\right)$ and measures directly related to aerobic power such as anaerobic threshold (TAn), field tests ${ }^{5}$ using steps of different heights ${ }^{6,7}$, using ergometers are highlighted ${ }^{8,9}$. On the other hand, stationary bicycles structured for a daily routine of collective physical training classes - known as spinning - could be used to measure aerobic fitness. However, there is an absence of precise intensity control ${ }^{10}$.

Among the most usual physiological measures related to exercise, there are those associated with lactacidemic responses, given their relevance in the prescription of exercise intensity in cyclic sport modalities with aerobic predominance ${ }^{11}$. These measures have also been con- sidered appropriate to identify exercise intensity ${ }^{12}$. TAn, minimum lactate, maximum stable lactate phase, and individual anaerobic threshold are procedures that evaluate, using the lactate concentration ([LAC]), the aerobic capacity $^{13}$. In addition to [LAC] analysis, heart rate (HR), heart rate deflection point (HRDP), maximum ventilatory deflection point ${ }^{14}$, ventilatory threshold ${ }^{15}$, oxygen uptake $\left(\mathrm{VO}_{2}\right)$, and rating of perceived exertion (RPE) are considered during incremental tests ${ }^{16}$. Furthermore, the first ventilatory threshold is recommended as a submaximal physiologic index and has been used to determine functional measures, prescription, and training intensity con$\operatorname{trol}^{17}$, mainly due to its non-invasive characteristic ${ }^{18}$.

Cycle ergometers are commonly used in conducting research and laboratory tests and are frequently used as equipment for aerobic power measurement and other physiologic responses associated with maximal effort as well ${ }^{19}$. Although researches with physical exercise prescriptions are well established, the viability of spinning bicycles to carry out maximal effort incremental tests (MEIT) is insufficiently investigated.

In this sense, this study aimed to investigate the concordance between an electromagnetic cycle ergometer of 
reference (Ergo-167) and a spinning bicycle (Keiser ${ }^{\mathrm{TM}}$ M3), for physiologic measures associated with a with maximal effort incremental test.

\section{Methods}

\section{Experimental approach to the problem}

For data collection, the participants visited the laboratory in two non-subsequent days, with a minimal interval of $72 \mathrm{~h}$. Before the visits, each participant was instructed not to consume any alcoholic beverages or stimulants (such as caffeine and tea) in the $24 \mathrm{~h}$ period before the tests, to sleep at least $8 \mathrm{hrs}$ the night before the visit, to be hydrated and adequately fed during the hours before the procedures, and solid meals were prohibited $60 \mathrm{~min}$ before the tests, only water ingestion was allowed in this period.

The samples were collected at the biochemistry and exercise physiology laboratory at the Federal University of Pelotas (UFPel) with controlled temperature and humidity at $21^{\circ}$ and $60 \%$, respectively, and in each session, the participants performed MEIT in different cycle ergometers in random order and counterbalanced. One of the cycle ergometers often used in incremental tests exhibits electromagnetic characteristic (Ergo-FIT ${ }^{\mathrm{TM}}$, Ergo-167 Cycle model, Pirmasens, German), and has been employed in evaluations of different groups, such as sedentary women ${ }^{20}$, diabetic subjects ${ }^{21}$ and, including, young people with obesity ${ }^{22}$. The other ergometer, KeiserTM M3 indoor Cycle (Keiser ${ }^{\mathrm{TM}}$, Chicago, USA), usually found in gyms and used for spinning classes, shows intensity manipulation from levers which present gears (from 1 to 24) associated with magnetic resistance on an LCD. In addition to estimating the power production based on rotations per minute (rpm), the Keiser ${ }^{\mathrm{TM}}$ M3 indoor Cycle presents similar ergonomics to the equipment used in cycling events ${ }^{14}$.

Subjects

This investigation is characterized as a validation study where fifteen women were enrolled with mean age, height, and weight of $19.2 \pm 4$ years, $169.6 \pm 7.6 \mathrm{~cm}$, and $61.7 \pm 10.1 \mathrm{~kg}$, respectively. The inclusion criteria of the participants were to be university students, not the practice of physical exercises systematically, not have experience with the use of cycle ergometers, and not on continuous therapy. After this phase, they read and signed the free and informed consent form (research project approved in the local ethics committee, protocol number 68577917.0.1001.5313).

\section{Procedures}

The heart rate was measured by using a Polar device (RS800CXTM, Kempele, Finland) to register the heart rate
(HR). The gas exchange analysis was carried out using a VO2000 analyzer, previously calibrated according to the manufacturer instructions, and the Breeze software (Medgraphics $^{\mathrm{TM}}$, Minnesota, USA), with a high flow pneumotachograph, neoprene ${ }^{\mathrm{TM}}$ medium size masks, with records of mean values for every three ventilations. The determination of blood lactate concentration ([LAC]) was performed using Yellow Spring Instruments (YSI) electrochemical analyzer, 2300 Sport model (Ohio, USA). RPE was collected with a 6-20 scale stit $^{16}$.

As baseline measures, the participants were instructed to sit for 10 minutes to be at resting heart rate $\left(\mathrm{HR}_{\text {rest }}\right)$, recording the mean value in $10 \mathrm{~min}^{24}$. Gas exchanges were collected until the last $5 \mathrm{~min}$ of the resting phase. At the end of this period, $15 \mu \mathrm{L}$ of blood was collected by a puncture on the fingertip, previously sterilized with $70 \%$ alcohol, using procedure gloves and disposable lancets (Softclick ${ }^{\circledR}$ ), which were transported to an Eppendorf tube containing $30 \mu \mathrm{L}$ of ethylenediamine tetraacetic acid (EDTA) for [LAC] analysis.

At the end of each stage and immediately at the end of the MEIT, HR, [LAC], oxygen uptake (VO2), and RPE data were collected and registered.

\section{Maximal Effort Incremental Test (MEIT)}

After familiarization with the cycle ergometers, description and explanation of the procedures and adjustments related to body position as well (seat height, seat distance, and handlebar on each cycle ergometer), standardized heating was performed by pedaling in a controlled cadence of 55 to $65 \mathrm{rpm}$ and using the same initial load of the tes $\mathrm{t}^{25}$. After warming up, not using the gas exchange, $4 \mathrm{~min}$ of recovery were granted, and then the VO2000 was installed during and after MEIT measurements.

To carry out MIET in both ergometers, each participant should pedal in a controlled cadence ${ }^{25}$ for two minutes at each stage. In the ERGO FIT (MEIT-EF), the procedure started with 50 watts $(\mathrm{W})$ of the initial load, and $25 \mathrm{~W}$ increments ${ }^{20}$, which occurred until the interruption of the test. In the Keiser ${ }^{\mathrm{TM}}$ M3 (MEIT-M3), the wattage is calculated as the product of speed (rpm) for the gear (from 1 to 24), considering the time required (in minutes) for its $16.8 \mathrm{~kg}$ wheel to travel one meter, and is presented on the display. A pilot study, carried out in our laboratory, identified that, for speed, varying between 55 and $65 \mathrm{rpm}$, two gear increments are associated with power production improvements near $25 \mathrm{~W}$. We would like to emphasize that during this pilot session, an independent sample $(\mathrm{n}=10)$ was used to measure the intraclass correlation coefficient between the test and retest of the Keiser ${ }^{\mathrm{TM}} \mathrm{M} 3$ ergometer (ICC $=0.854)$, considering the $\mathrm{VO} 2 \mathrm{max}$ as a variable. Thus, MEIT-M3 started with the ergometer in the fourth gear, and the progression occurred with increments of two gears every two minutes until its interruption. The MEIT interruptions occurred when the participant: i) reached 
voluntary fatigue, losing the ability to keep the minimum established cadence; reported not being able to finish the subsequent stage; or iii) presented with sudden onset, identified by cyanosis, pallor or reported malaise. Exhaustion was verified using the result from the RPE.

\section{Physiological parameters associated with MEIT}

Different physiologic values were obtained using concordance measurements between the cycle ergometers. Among them, blood ([LAC]), ventilatory (VO2), and cardiac (HR) variables were considered to monitor the responses in both bicycles. Blood measurement for anaerobic threshold analysis considered Onset of Blood Lactate Accumulation (OBLA), assuming the beginning of [LAC] accumulation tends to increase abruptly, and the point of change was not analyzed based on a fixed number (4mmol), but at the moment of a relevant change in the [LAC] curve [26], identified by scatter plot. In addition, the peak lactate $\left([\mathrm{LAC}]_{\text {peak }}\right)$ value, that is, the highest value of $[\mathrm{LAC}]$ obtained in the MEIT, was also considered.

Regarding the ventilatory component, the visual inspection method was used to determine VO2TAn, where the deviation point was inspected and defined by two researchers and, in case of divergence, the participation of a third auxiliary researcher was requested ${ }^{15}$. A previous study found a strong association between $\mathrm{VO} 2$, wattage, $\mathrm{HR}$, and ventilatory threshold, the latter being a non-invasive measurement, and a facilitator for thresholds identification ${ }^{15}$. Relative $\mathrm{VO}_{2 \max }(\mathrm{mL} / \mathrm{kg} / \mathrm{min})$ values were also considered.

In respect to HR, from de immediate recording of each beat, a graphic line was observed, and with the increase of loads, the discontinuity point of the line was identified, indicating the anaerobic threshold (HRTAn) ${ }^{27}$. This threshold is indicated to occur when, during exercise, the individual reaches $\mathrm{VO} 2$ between 50 and $60 \%$ of the $\mathrm{VO} 2 \mathrm{max}$, since there is a decrease in the vagal activity, and there is a predominance of the sympathetic nervous system which indicates the respective physiological threshold $^{26}$, which tends to coincide with the first lactate threshold. Also, HRTAn, HRmean, and HRmax were considered during the test. In order to identify these HR markers, data recorded on the cardio-frequency meter were used, which were downloaded and analyzed using the Polar ProTrainer software (Kempele, Finland). Subsequently, the generated numbers were plotted and the HRTAn was identified using the HRDP method, which is the maximum point of distance from a curve described by the ventilatory variation values, with a straight line drawn by the start and endpoints of that curve ${ }^{14}$.

\section{Statistical analysis}

Firstly, descriptive data are shown as mean and standard deviation, and the comparisons between means of physiologic variables were performed with the paired Ttest. Lin's test was used to measure the correlations, and Bland-Altman analysis was employed to measure the concordance among variables. The statistical routines were conducted with GraphPad Prism 7 (San Diego, California, EUA) and Stata 12 (Timbarlake, UK) software systems. A $5 \%$ significance level was adopted.

\section{Results}

Descriptive data, according to the ergometer and the group of variables, as well as the percentage of technical error measurement (TEM) are shown in Table 1. The Paired T-test did not identify differences in variables related to RPETAn $(\mathrm{p}=0.609)$, HRTAn $(\mathrm{p}=0.120)$ and [LAC]TAn $(p=0.728)$. The same occurs when the maximum responses are considered at the end of the test, being $\operatorname{RPEmax}(\mathrm{p}=0.371), \mathrm{VO}_{2 \max }(\mathrm{p}=0.410)$, [LAC]peak $(p=0.371)$ and effort time $(p=0.096)$.

Table 2 presents bias and standard deviation (sd), as well as limits of agreement (LoA), both obtained through

Table 1 - Descriptive data of the sample, considering each ergometer $(\mathrm{n}=15)$.

\begin{tabular}{|c|c|c|c|c|}
\hline Variables & Keiser-M3 & Ergo-167 & $t$ (p-value) & Technical error of measurement \\
\hline \multicolumn{5}{|c|}{ Anaerobic threshlod measures } \\
\hline RPE (u.a.) & $14 \pm 2$ & $13 \pm 3$ & $\mathrm{t}=0.523(\mathrm{p}=0.609)$ & $6.76 \%$ \\
\hline HR (bpm) & $146 \pm 14.44$ & $149.8 \pm 9.41$ & $\mathrm{t}=-1.656(\mathrm{p}=0.120)$ & $2.6 \%$ \\
\hline $\mathrm{VO}_{2}(\mathrm{~mL} / \mathrm{kg} / \mathrm{min})$ & $14.62 \pm 3.25$ & $15.14 \pm 2.70$ & $\mathrm{t}=2.247(\mathrm{p}=0.041)$ & $3.55 \%$ \\
\hline$[\mathrm{LAC}](\mathrm{mmol} / \mathrm{L})$ & $3.26 \pm 149$ & $3.53 \pm 1.50$ & $\mathrm{t}=-0.354(\mathrm{p}=0.728)$ & $8.28 \%$ \\
\hline \multicolumn{5}{|c|}{ Measures at the end of the test } \\
\hline $\mathrm{RPE}_{\max }$ (u.a.) & $19.66 \pm 2.76$ & $18.92 \pm 1.20$ & $\mathrm{t}=0.24(\mathrm{p}=0.371)$ & $3.91 \%$ \\
\hline $\mathrm{HR}_{\max }(\mathrm{bpm})$ & $186 \pm 53$ & $188.4 \pm 11.83$ & $\mathrm{t}=-2.102(\mathrm{p}=0.719)$ & $1.39 \%$ \\
\hline $\mathrm{VO}_{2 \max }(\mathrm{mL} / \mathrm{kg} / \mathrm{min})$ & $38.37 \pm 6.97$ & $36.04 \pm 8.40$ & $\mathrm{t}=2.247(\mathrm{p}=0.410)$ & $6.4 \%$ \\
\hline$[\mathrm{LAC}]_{\mathrm{pico}}(\mathrm{mmol} / \mathrm{L})$ & $9.97 \pm 2.51$ & $9.71 \pm 2.56$ & $\mathrm{t}=0.924(\mathrm{p}=0.371)$ & $2.67 \%$ \\
\hline Effort time (min) & $11.2 \pm 2.11$ & $9.86 \pm 2.81$ & $\mathrm{t}=1.784(\mathrm{p}=0.096)$ & $13.59 \%$ \\
\hline
\end{tabular}

$\mathrm{RPE}=$ Rated Perceived Exertion; $\mathrm{HR}=$ Heart Rate; $\mathrm{VO}_{2}=$ oxygen uptake; $[\mathrm{LAC}]=$ lactate concentration; $[\mathrm{LAC}]_{\text {peak }}=$ lactate concentration peak. 
Table 2 - Concordance of Bland-Altman and Lin's correlation between ergometers $(n=15)$.

\begin{tabular}{lcc}
\hline Variables & Concordance of Bland-Altman mean difference \pm sd (IC95\%) & Lin's correlation r (classification) \\
\hline Anaerobic threshlod measures & $0.33 \pm 2.469(-4.51-5.17)$ & 0.17 (very weak) \\
$\mathrm{RPE}($ u.a.) & $-3.8 \pm 8.89(-21.22-13.62)$ & 0.76 (Strong) \\
$\mathrm{HR}(\mathrm{bpm})$ & $-0.53 \pm 2.93(-6.27-5.22)$ & 0.59 (Moderate) \\
$\mathrm{VO}_{2}(\mathrm{~mL} / \mathrm{kg} / \mathrm{min})$ & $-0.19 \pm 1.576(-3.28-2.89)$ & 0.42 (Moderate) \\
{$[\mathrm{LAC}](\mathrm{mmol} / \mathrm{L})$} & & 0.23 (very weak) \\
Measures at the end of the test & $-0.33 \pm 1.397(-3.07-2.40)$ & 0.72 (Strong) \\
$\mathrm{RPE}_{\max }($ u.a.) & $-1.86 \pm 8.08(-17.72-13.98)$ & 0.81 (Strong) \\
$\mathrm{HR}_{\max }(\mathrm{bpm})$ & $2.33 \pm 4.25(-6.01-10.67)$ & 0.82 (Strong) \\
$\mathrm{VO}_{2 \max }(\mathrm{mL} / \mathrm{kg} / \mathrm{min})$ & $0.26 \pm 1.50(-2.68-3.20)$ & \\
{$[\mathrm{LAC}]_{\text {pico }}(\mathrm{mmol} / \mathrm{L})$} &
\end{tabular}

$\mathrm{RPE}=$ Rated Perceived Exertion; $\mathrm{HR}=$ Heart Rate; $\mathrm{VO}_{2}=$ oxygen uptake; [LAC] = lactate concentration; [LAC $]_{\text {peak }}=$ lactate concentration peak.

the Bland-Altman analysis (2000). Moreover, $r$ values and the respective classifications are presented. A concordance was identified regarding the classification between HRTAn $(\mathrm{r}=0.762)$ and HRmax $(\mathrm{r}=0.719)$, which identified a strong correlation between cardiac variables. The lactacidemic responses during ([LAC]TAn $r=0.242$ ) showed moderate correlation; however, [LAC]peak $(r=0.820)$ showed a strong correlation between ergometers. As well as blood responses, the threshold during MEIT showed a moderate correlation $(r=0.597)$. Nevertheless, a strong correlation was verified between ergometers in VO2max $(r=0.809)$. About RPE at the anaerobic threshold, there was a very low correlation between the ergometers (RPE$\mathrm{TAn}=0.176$ ), although a relatively small mean difference $(0.33 \pm 2.47)$ was obtained in the Bland-Altman analysis.

\section{Discussion}

This investigation aimed to validate the Keiser ${ }^{\mathrm{TM}}$ cycle ergometer, M3 model, for physiologic evaluation in MEIT, comparing it with a previously validated cycle ergometer (Ergo-FITTM, Ergo-167 Cycle model). In this sense, the main findings indicate the following: i) there is a concordance between cardiac results obtained from HRmax and in the anaerobic threshold identification using HR during the test; ii) there is a strong concordance between the ergometers for [LAC]peak variable; iii) a strong correlation between bicycles used for VO2max measurements was found; iv) the MEIT protocol, elaborated for tests in Keiser ergometer, is efficient when the aim is maximal physiologic measurements of HR, [LAC], VO2 and RPE.

Among the measured physiological parameters, there is a strong agreement between ergometers for HRmax $(r=0.719)$, which is defined as the highest HR value that an individual can achieve in maximal effort until exhaustion is reached ${ }^{28}$. In addition, an important indicator was considered to quantify maximum efforts during the test in ergometers ${ }^{9}$, and the HRmax is widely used to aerobic exercise intensity prescription and is directly related to the VO2 $\max ^{29}$. HR increased in values according to the load progression in MEIT, and this response was similar in both considered instruments. The mean difference between ergometers for $\mathrm{HR}_{\max }$ in this study was $-1.86 \pm 8.08 \mathrm{bpm}$, which corroborates with the data observed in the study of Silva ${ }^{18}$, in which MEIT's were carried out using different cycle ergometers and, although a lower/upper difference was identified (182.05 $\pm 12.39 \mathrm{bpm}$ e $180.57 \pm 12.58 \mathrm{bpm}$ ), no significant differences were observed in this cardiac parameter $(p=0.127)$.

Regarding submaximal measures, the results of this investigation agree with those obtained by Grazzi et al. ${ }^{31}$, which verified concurrence in the points of deflection of the HR in different TIMS, showing similar linearity. The concordance between studies can be justified as both studies used test protocols with small increments in fixedtime stages, which follows the recommendations of Conconi et al. ${ }^{32}$. In addition, the values obtained on the HRTAn showed a strong correlation $(\mathrm{r}=0.76)$. This finding is extremely important, as suggested by Grassl ${ }^{38}$ since individuals with similar values of cardiorespiratory capacity presented different physiological responses during acute exercise performed in the same percentage concerning $\mathrm{VO} 2$ max.

The VO2max is described as the higher rate of oxygen captured, transported, and used by the muscle during exhaustive work, and this variable is also used to describe cardiovascular and respiratory of oxygen transport capacity, making it one of the best indicators of cardiorespiratory function ${ }^{33,4}$. In the present study, VO2max showed a high correlation between ergometers $(r=0.809)$, with a mean difference of only $2.33 \mathrm{~mL} / \mathrm{kg} / \mathrm{min}$. Thus, it is possible to assume that the measurement of $\mathrm{VO} 2 \mathrm{max}$ using the Keiser-M3 ergometer is accurate in relation to the Ergo-167 ergometer. These results are relevant, considering that VO2max is one of the most essential mea- 
TAn Rated Perceived Exertion

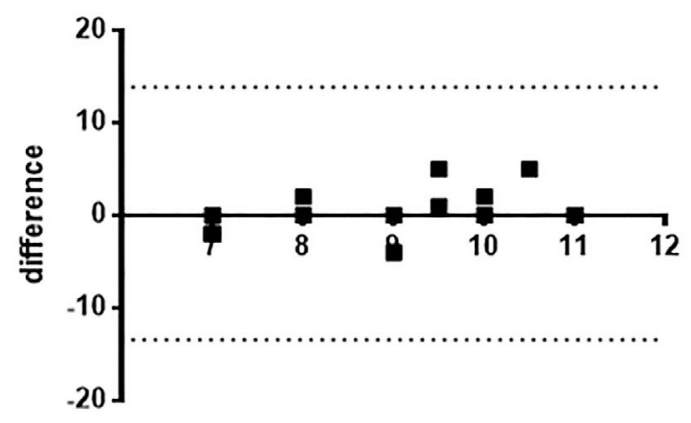

TAn Oxygen Uptake

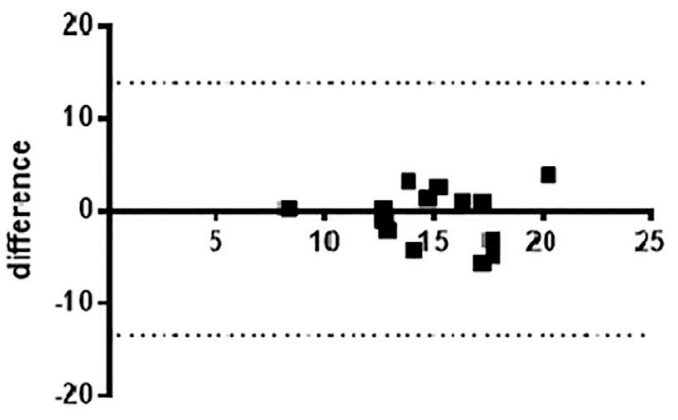

average

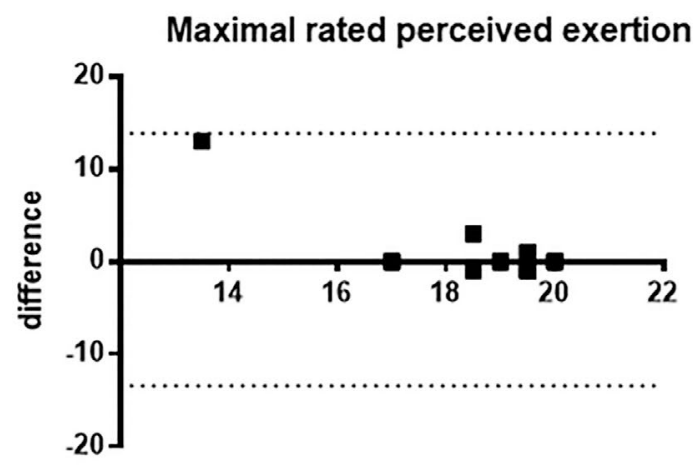

average

Maximal oxygen uptake

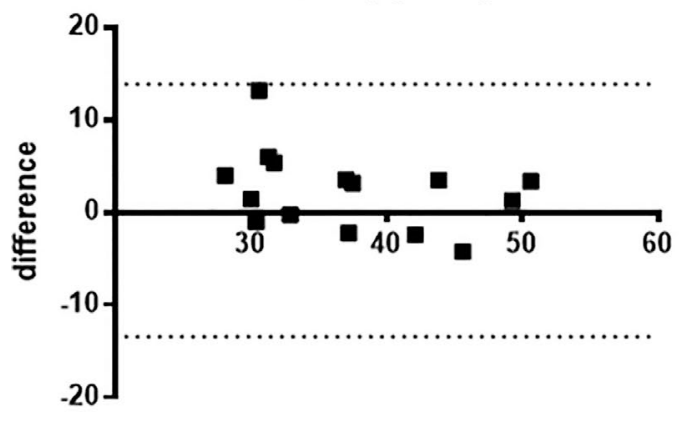

average
TAn Heart Rate

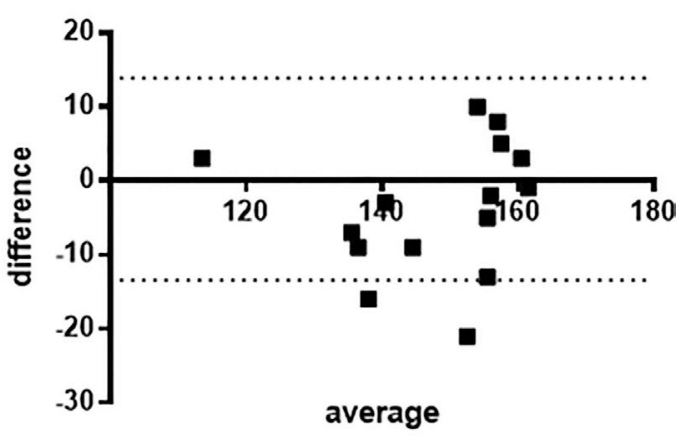

TAn Lactate

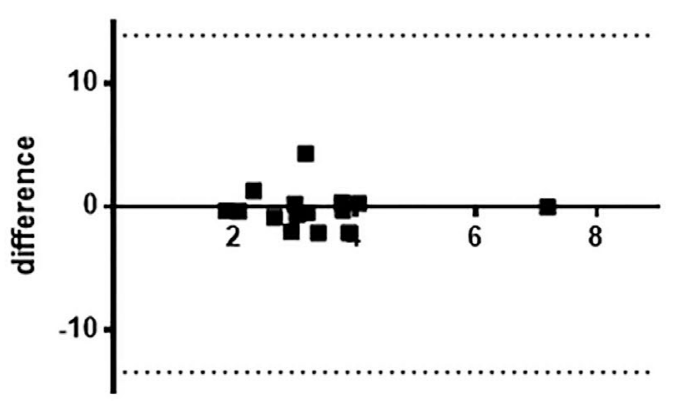

average
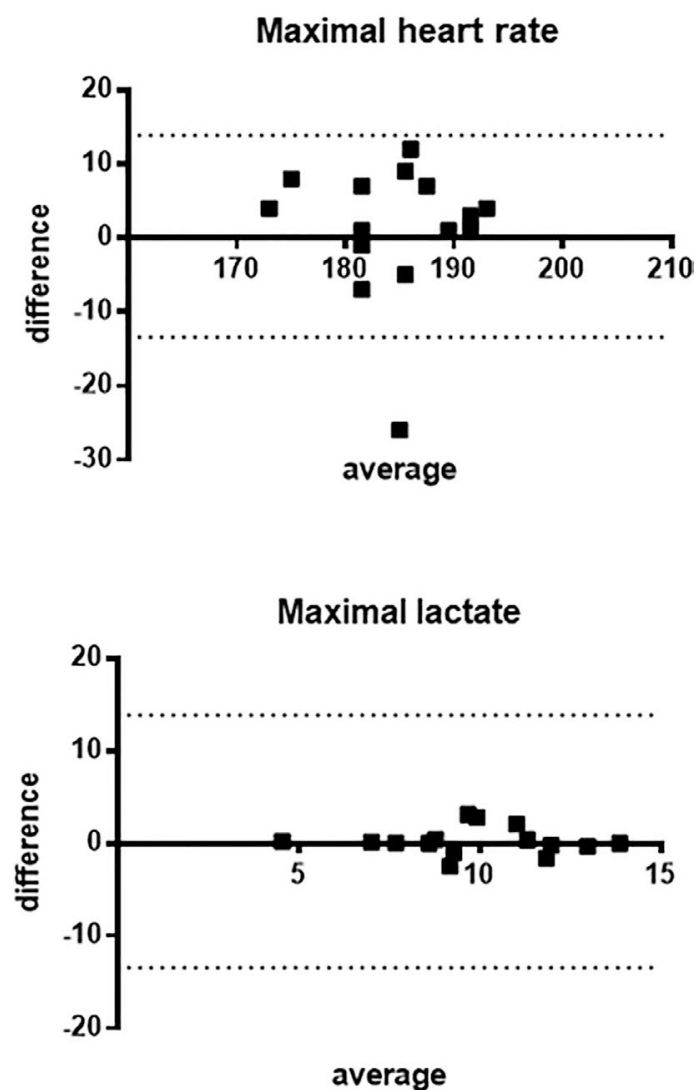

Figure 1 - Bland-Altman graphs for agreement between ergometers. 
sures in the exercise physiology area, mainly if they are analyzed for maximum aerobic power and aerobic exercise prescription $^{30}$. Barbinau et al. ${ }^{34}$ found that there may be a difference between the same test and retest gas analysis system, suggesting that tolerable differences for VO2max in repeated measurements may reach $4 \%$. In addition, Hodges et al. ${ }^{35}$ stated that changes between 3$10 \%$ are acceptable and have been cited in the literature. Yule et al. ${ }^{36}$ reported differences of $15 \%$ between three equal gas analyzers but belonging to different laboratories. Considering the obtained data from the same gas analyzer during the collections, a variation of $3.5 \%$ was found, that is, lower than the variation indicated in the aforementioned studies. Moreover, regarding VO2TAn values, despite showing statistical difference $(p=0.041)$, the correlation test found moderate values $(\mathrm{r}=0.59)$ between ergometers. Also, the Bland-Altman concordance test demonstrated that the values obtained are within the limits of the agreement provided by the analysis. In a study by Hirakata $^{39}$, it was indicated that these measures of agreement and correlation may have limitations when used in isolation, highlighting the need for complementary use between them. Thus, observing the results provided by the statistical treatment, it is possible to highlight the feasibility of using Keiser-M3 for VO2TAn measurements.

Regarding [LAC], its kinetics exhibits two distinct post-effort phases, one when there is a rate of increase and another when there is a rate of decrease ${ }^{37}$. When considering the [LAC]peak, the obtained results show strong concordance $(r=0.820)$ between the compared instruments, showing its usefulness for the realization of maximum efforts tests. This accumulation in [LAC] can be evidenced by the high energy required by the anaerobic system, which increases blood rates of this metabolic product $^{13}$. Gross et al. ${ }^{38}$, when investigating cyclists, found that all subjects, even when they did not have a maximal stable phase of [LAC], on reaching fatigue, concomitantly presented similar maximum values of [LAC], making the findings of the present study valid. This investigation also verified the applicability and consistency of the mathematical model HRDP on the identification of the anaerobic threshold. It is also accepted that the MEIT protocol used in the course of this study becomes replicable since the organic responses induced in the two tests were comparable. Another vital point among ergometers is comfort. According to the women participating in this research, the Ergo-167 is uncomfortable and encourages interruption of the test, while the Keiser-M3 is more similar to a conventional bicycle, making it the preferred bicycle among participants. Regarding VO2TAn values, no statistical differences were found $(\mathrm{p}=0.728)$ between MEIT's, with a moderate correlation $(\mathrm{r}=0.42)$ and an average difference of $0.2 \mathrm{mmol} / \mathrm{L}$ between tests. In a study conducted by Okano et al. ${ }^{15}$, it was possible to find interindividual variation between 1.9 and $5.04 \mathrm{mmol} / \mathrm{L}$, high- lighting the feasibility of using the Keiser-M3 ergometer in conjunction with the proposed protocol for MEIT. The difference between the values of the studies may be associated with the fact that the authors of the previously mentioned study used frozen samples for analysis, whereas the samples used in this study were immediately analyzed after collection.

Based on the findings of the present study, it was observed that parameters about RPE presented low reproducibility, although they did not present differences between averages. On the other hand, HRTAn, HRmax, VO2max, and [LAC]peak showed a high level of agreement between ergometers. Thus, the reproducibility of using the Keiser stationary bicycle, model M3, for the realization of MEIT is highlighted, considering that both ergometers may cause physiological stress of similar magnitude for this sample.

The findings in this study are relevant since accurate assessments, both in laboratories and in gyms, are necessary for the planning and monitoring of physical exercise programs. This study demonstrates that stationary bicycles, easily found in different locations, laboratories, or not, may provide a reliable analysis of parameters obtained through MEIT. In the present study, a high correlation was found between the maximum variables and the use of Keiser-M3. Therefore, when the focus of the incremental test is maximum parameters, the Keiser-M3 cycle ergometer is an efficient alternative instrument, without compromising data obtained at the end of the session.

\section{References}

1. Noakes TD. Physiological models to understand exercise fatigue and the adaptations that predict or enhance athletic performance. Scand J Med Sci Sports. 2000;10: 123-145.

2. Christensen J, Tang L, Doherty P, Langhorn C, Langberg H. Test-retest reliability of a maximal arm cycle exercise test for younger individuals with traumatic lower-limb amputations. Eur. J. Physiother. 2019;21:1-6.

3. Whibley J, Peters CJ, Halliday LJ, Chaudry AM, Allum WH. Poor performance in incremental shuttle walk and cardiopulmonary exercise testing predicts poor overall survival for patients undergoing esophagogastric resection. Eur J Surg Oncol. 2018;44(5): 594-599.

4. American College of Sports Medicine. Position stand on progression models in resistance exercise for healthy adults. Med Sci Sports Exerc. 2009;41:687-708.

5. Bangsbo J, Iaia FM, Krustrup P. The Yo-Yo intermittent recovery test. Sports med. 2008;38(1): 37-51.

6. Looyestyn J, Kernot J, Boshoff K, Maher C. A web-based, social networking beginners' running intervention for adults aged 18 to 50 years delivered via a Facebook group: a randomized controlled trial. J Med Internet Res. 2018;20(2): e67.

7. Laroche D, Mangin M, Besson D, Naaim A, Gouteron A, Casillas JM. A number of raised steps: A tool to assess brief 
and intense effort involving anaerobic metabolism. Ann Phys Rehabil Med. 2018;61(3):156-163.

8. Boscolo VF, Ferreira JLM. Mixed martial arts: Rotinas de condicionamento e avaliação da aptidão física de lutadores de Pelotas/RS. Rev bras ciênc esporte. 2013;35(3):611-626.

9. Bandyopadhyay A. Validity of Cooper's 12-minute run test for estimation of maximum oxygen uptake in male university students. Biol Sport. 2015;32(1):59-63.

10. Shepherd SO, Wilson OJ, Taylor AS, Thøgersen-Ntoumani C, Adlan AM, Wagenmakers AJM, et al. Low-Volume High-Intensity Interval Training in a Gym Setting Improves Cardio-Metabolic and Psychological Health. PLoS ONE. 2015;10(9):1-17.

11. Laursen PB, Rhodes EC, Langill RH, McKenzie DC, Taunton JE. Relationship of exercise test variables to cycling performance in an Ironman triathlon. Eur J Appl Physiol. 2002;87:433-40.

12. Castagna C, D'Ottavio S, Cappelli S, Póvoas SCA. The Effects of Long Sprint Ability Oriented Small-Sided Games Using Different Players-to-Pitch Area on Internal and External Load in Soccer Players. Int J Sports Physiol Perform. 2019;12:1-23.

13. Dragonea P, Zacharakis E, Kounalakis S, Kostopoulos N1, Bolatoglou T1, Apostolidis N. Determination of the exercise intensity corresponding with maximal lactate steady state in high-level basketball players. Res Sports Med. 2019;27(1):112-120.

14. Delevatti RS, Kanitz AC, Alberton CL, Pantoja PD, Marson EC, Pinho CDF, et al. Heart rate deflection point as an alternative method to identify the anaerobic threshold in patients with type 2 diabetes. Apunts Med Sport. 2015;50(188):123128.

15. Okano AH, Altimari LR, Simões HG, Moraes AC, Nakamura FY, Cyrino ES, et al. Comparison between anaerobic threshold determined by ventilatory variables and blood lactate response in cyclists. Rev Bras Med Sport. 2006;12 (01):39-44.

16. Borg GAV. Physiological bases of perceived exertion. Med Sci Sports Exerc. 1982;14(3):377-387.

17. Neufeld EV, Wadowski J, Boland DM, Dolezal BA, Cooper CB. Heart Rate Acquisition and Threshold-Based Training Increases Oxygen Uptake at Metabolic Threshold in Triathletes: A Pilot Study. Int J Exerc Sci. 2019;12(2):144-154.

18. Silva CGDS, Castro CLBD, Franca J, Bottino A, Myers J, Araujo CGS. Cardiorespiratory optimal point in professional soccer players: a novel submaximal variable during exercise. Int. J. Cardiovasc. Sci. 2018;31(4):323-332.

19. Siqueira GDJ, Maioni LL, Soares V, Lima WA. Subacute hypotensor effect of a high-intensity interval training session. Cinergis. 2017;18(2):114-120.

20. Nasci ABA, Orcy RB, Cabistany LD, Formalioni A, Vecchio FBD. Acute responses of high-intensity circuit training in women: low levels of physical fitness show greater muscle damage. Rev Bras Cineantropom Desempenho Hum. 2018;20 (5):391-401.

21. Ferraresi C, Baldissera V, Perez SE, Junio EM, Bagnato VS, Parizotto NA. One-repetition maximum test and isokinetic leg extension and flexion: Correlations and predicted values. Isokinetic Exerc Sci. 2013;21(1):69-76.
22. Bertoli J, Diefenthaeler F, Detanico D, et al. Can mat Pilates intervention increase lower limb rate of force development in overweight physically active older women? Sport Sci Health. 2019;15(1): 1-9.

23. Soriano-Maldonado A, Romero L, Femia P. A learning protocol improves the validity of the Borg 6-20 RPE scale during indoor cycling. Int J Sports Med. 2014;35(5):379-84.

24. Barth J, Del Vecchio FB. Effects of ventilatory frequency on heart rate variability indexes. Rev Iberoa Arritmología. 2014;5(1): 185-193.

25. Vercruyssen F, Missenard O, Brisswalter J. Relation between the slow component of oxygen uptake and the surface EMG during heavy exercise in humans: Influence of the frequency of pedaling. J Electromyogr. Cinesiol. 2009;19(4): 676-684.

26. Garcia-Tabar I, Gorostiaga EMA. "Blood relationship" between the overlooked minimum lactate equivalent and maximal lactate steady state in trained runners. back to the old days?. Front Physiol. 2018;9:1-13.

27. Guimarães MP, Silva S. Comparison between critical velocity and speed at the anaerobic threshold of moderately trained 5-km runners. J Phys Educ Sport. 2017;(4):24372442.

28. Durmic T, Đjelic M, Gavrilovic T, Antic M, Jeremic R, Vujovic A, et al. Usefulness of heart rate recovery parameters to monitor cardiovascular adaptation in elite athletes: The impact of the type of sport. Physiol Int. 2019;106(1):114.

29. American College of Sports Medicine (ACSM). Guidelines of exercise testing and exercise prescription. 8th ed. Philadelphia: Lea \& Febiger, 2009.

30. Silva RAS. Validation of an indoor cycling ergometer bicycle. Master's thesis, Universidade Católica de Brasília, Brasília, 2008.

31. Grazzi G, Casoni I, Mazzoni G, Uliari S, Conconi F. Protocol for the Conconi test and determination of the heart rate deflection point. Physiolol Res. 2005;54(4): 473-475.

32. Conconi F, Grazzi G, Casoniet al. The Conconi test: methodology after 12 years of application. Int J Sports Med. 1996;17(07):509-519.

33. Tucker WJ, Angadi SS, Gaesser GA. Excess postexercise oxygen consumption after high-intensity and sprint interval exercise, and continuous steady-state exercise. J Strength Cond Res. 2016;30(11):3090-97.

34. Babineau C, Léger L, Long A, Bosquet L. Variability of maximum oxygen consumption measurement in various metabolic systems. J Strength Cond Res. 1999;13(4):318324.

35. Hodges LD, Brodie DA, Bromley PD. Validity and reliability of selected commercially available metabolic analyzer systems. Scand J Med Sci sports. 2005;15(5): 271-279.

36. Yule ET. Inter-laboratory reliability of VO?max and submaximal measurements. Master's thesis, Ball State University, Municie. 1995.

37. Bret C, Messonnier L, Nouck JN, Freund H, Dufour AB, Lacour JR. Differences in lactate exchange and removal abilities in athletes specialised in different track running events (100 to 1500 m). Int J Sports Med. 2003;24(02):108113. 
38. Gross1 T, Barbosa LF, Lucas RD, Guglielmo LGA. Oxygen consumption during cycling in the maximal stable phase of blood lactate until exhaustion: continuous vs. continuous model intermittent. Rev Andal Med Deporte. 2013;7 (4):155-161.

39. Denison HJ, Syddall HE, Dodds R, Martin HJ, Finucane FM, Griffin SJ, et al. Effects of Aerobic Exercise on Muscle Strength and Physical Performance in Community-dwelling Older People from the Hertfordshire Cohort Study: A Randomized Controlled Trial. J Am Geriatr Soc. 2013;61:10346.

\section{Corresponding author}

Rousseau Silva da Veiga UFPEL, Escola Superior de
Educação Física R. Luís de Camões, 625, Três Vendas Pelotas Rio Grande do Sul, Brasil.

E-mail: rousseauveiga@gmail.com

Manuscript received on October 31, 2019

Manuscript accepted on April 28, 2020

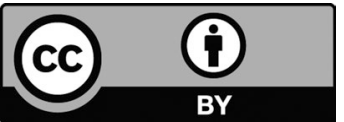

Motriz. The Journal of Physical Education. UNESP. Rio Claro, SP, Brazil - eISSN: 1980-6574 - under a license Creative Commons - Version 4.0 